\title{
Constructive updating/downdating of oblique projectors: a generalization of the Gram-Schmidt process
}

\author{
Laura Rebollo-Neira \\ Aston University, Birmingham B4 7ET, UK
}

\begin{abstract}
A generalization of the Gram-Schmidt procedure is achieved by providing equations for updating and downdating oblique projectors. The work is motivated by the problem of adaptive signal representation outside the orthogonal basis setting. The proposed techniques are shown to be relevant to the problem of discriminating signals produced by different phenomena when the order of the signal model needs to be adjusted.
\end{abstract}

\section{Introduction}

An operator $\mathcal{O}$ is a projector if it is idempotent, i.e., if it is endowed with property $\mathcal{O}^{2}=\mathcal{O}$. The projection is along (or parallel to) its null space $\mathcal{N}(\mathcal{O})$ and onto its range $\mathcal{R}(\mathcal{O})$. This entails that $\mathcal{O} v=v$ for $v \in \mathcal{R}(\mathcal{O})$ and $\mathcal{O} v=0$ for $v \in \mathcal{N}(\mathcal{O})$. If the subspaces $\mathcal{R}(\mathcal{O})$ and $\mathcal{N}(\mathcal{O})$ are orthogonal the operator is called an orthogonal projector, which is the case if and only if $\mathcal{O}$ is self-adjoint. Otherwise it is called oblique projector.

Oblique projectors, though introduced early $[1,2]$, have received less attention than orthogonal projectors. Nevertheless, quite recently there has been a renewed interest in relation to their properties and applications [3-12]. In particular, oblique projectors have been shown to be of significant relevance to signal processing techniques [13-16]. The present effort is very much motivated by problems arising in the area of signal representation outside the traditional orthogonal basis setting [17-20]. In such a context a signal $f$, represented mathematically as an element of a vector space, is approximated as a linear expansion of the form

$$
f^{k}=\sum_{i=1}^{k} c_{i} v_{i} .
$$

The vectors $v_{i}$ in (11) are sometimes sequentially fed or chosen according to some optimality criterion. In such situations one needs to be in a position to effectively adapt the coefficients of the linear superposition so as to account for the possibility of changes in the model. This may entail a)increasing the order $k$ of the model by incorporating new terms in the expansion b)reducing the order by eliminating some terms in the expansion c)replacing some of the vectors in (11) by different ones.

Assuming that the signal space is an inner product space, for $f^{k}$ given in (1) to be the best approximation of a signal $f$ in a minimum distance sense, the coefficients in (1) should be calculated in such a way that $f^{k}$ is the orthogonal projection of $f$ onto $\operatorname{span}\left\{v_{i}\right\}_{i=1}^{k}$. This is the main reason for the popularity of orthogonal projectors in the context of approximation 
techniques. Nevertheless, suppose that the observed signal is produced by the interference of two phenomena so that the model (1) becomes

$$
\sum_{i=1}^{k} c_{i} v_{i}+\sum_{i=1}^{n} d_{i} w_{i}
$$

If one were interested in discriminating the phenomena by splitting the signal, the component in $\operatorname{span}\left\{v_{i}\right\}_{i=1}^{k}$ could be obtained by an oblique projection operation mapping the other component to zero. There is a broad range of applications in which this procedure happens to be of assistance [13]. Thus, we felt motivated to find recursive equations for adapting oblique projectors. Some of the equations to be proposed here are inspired by our previous work on recursive biorthogonalization for orthogonal projectors representation $[21,22]$. We have recently been made aware that such a work is closely related to earlier one on recursive generalized inverses $[23-26]$.

In spite of the fact that for most numerical implementations a projector is represented by a matrix, we prefer to think of projectors as operators acting by performing inner products. An important reason for this choice is the following: The equations can thereby be applied in general inner product spaces and comprise two very important cases in particular. Namely, the Euclidean inner product space, where a projector is indeed a matrix, and the space of functions of finite 2-norm. We like to see the proposed recursive equations as generalized Gram-Schmidt like procedures for generating sequences in inner product spaces. Such sequences give rise to oblique projectors onto nested subspaces and, of course, to orthogonal projectors as special case.

The paper is organized as follows: Section 2 introduces the notation along with a discussion on the general construction of oblique projectors. Section 3 provides the recursive equations for stepwise updating/downdating of such projectors. Applications are illustrated in Section 4 by i)recovering a simulated X-ray diffraction peak from a background and ii)filtering impulsive noise from the register of the motion of a system consisting of the superposition of damped harmonic oscillators. The conclusions are drawn in Section 5.

\section{Oblique projectors}

As already mentioned we will work in a general inner product space $\mathcal{H}$, where the square norm $\|.\|^{2}$ is induced by the inner product that we represent as $\langle\cdot, \cdot\rangle$. Given two closed subspaces, $\mathcal{V} \in \mathcal{H}$ and $\mathcal{W}^{\perp} \in \mathcal{H}$, such that $\mathcal{H}=\mathcal{V}+\mathcal{W}^{\perp}$ and $\mathcal{V} \cap \mathcal{W}^{\perp}=\{0\}$, the oblique projector operator onto $\mathcal{V}$ along $\mathcal{W}^{\perp}$ will be represented as $\hat{E}_{\mathcal{V} \mathcal{W}^{\perp}}$. Then $\hat{E}_{\mathcal{V} \mathcal{W}^{\perp}}$ satisfies:

$$
\begin{aligned}
\hat{E}_{\mathcal{V} \mathcal{W}^{\perp}}^{2} & =\hat{E}_{\mathcal{V} \mathcal{W}^{\perp}} \\
\hat{E}_{\mathcal{V W}}{ }^{\perp} v & =v, \quad \text { for any } \quad v \in \mathcal{V} \\
\hat{E}_{\mathcal{V W}} \mathcal{W}^{\perp} & =0, \quad \text { for any } \quad w \in \mathcal{W}^{\perp} .
\end{aligned}
$$

In the particular situation in which $\mathcal{W}^{\perp}$ happens to be the orthogonal complement of $\mathcal{V}$ in $\mathcal{H}$, i.e. if $\mathcal{W}^{\perp}=\mathcal{V}^{\perp}$, the operator is self-adjoint and represents an orthogonal projection onto $\mathcal{V}$. We emphasize this special case by using the particular notation $\hat{E}_{\mathcal{V} \mathcal{V}^{\perp}}=\hat{P}_{\mathcal{V}}$. In the sequel the orthogonal projector operator onto a subspace, say the subspace $\mathcal{X}$, will be indicated as $\hat{P}_{\mathcal{X}}$.

Let us assume that in general $\mathcal{V}=\operatorname{span}\left\{v_{i}\right\}_{i=1}^{k}$ and $\mathcal{W}=\operatorname{span}\left\{u_{i}\right\}_{i=1}^{k}$, with $\mathcal{W}$ the orthogonal complement of $\mathcal{W}^{\perp}$. Denoting as $\boldsymbol{e}_{i}, i=1, \ldots, k$ the standard orthonormal basis in $\mathbb{C}^{k}$, i.e., 
the inner product $\left\langle\boldsymbol{e}_{i}, \boldsymbol{e}_{j}\right\rangle=\delta_{i, j}$ with $\delta_{i, j}$ equal one if $i=j$ and zero otherwise, we define the operators $\hat{V}$ and $\hat{W}$ as

$$
\hat{V}=\sum_{i=1}^{k} v_{i}\left\langle\boldsymbol{e}_{i}, \cdot\right\rangle, \quad \hat{W}=\sum_{i=1}^{k} u_{i}\left\langle\boldsymbol{e}_{i}, \cdot\right\rangle .
$$

Thus the corresponding adjoint operators $\hat{W}^{*}$ and $\hat{V}^{*}$ are

$$
\hat{V}^{*}=\sum_{i=1}^{k} \boldsymbol{e}_{i}\left\langle v_{i}, \cdot\right\rangle, \quad \hat{W}^{*}=\sum_{i=1}^{k} \boldsymbol{e}_{i}\left\langle u_{i}, \cdot\right\rangle .
$$

The operations $\left\langle v_{i}, \cdot\right\rangle$ and $\left\langle u_{i}, \cdot\right\rangle$ indicate that $\hat{V}^{*}$ and $\hat{W}^{*}$ act by performing inner products in $\mathcal{H}$. The inner product is defined in such a way that for $f \in \mathcal{H}$ and $c$ a complex constant the mapping $\hat{V}^{*} c f$ produces a vector in $\mathbb{C}^{k}$ of the form $\hat{V}^{*} c f=c \sum_{i=1}^{k} \boldsymbol{e}_{i}\left\langle v_{i}, f\right\rangle$. The operation $\left\langle\boldsymbol{e}_{i}, \cdot\right\rangle$ indicates the inner product in $\mathbb{C}^{k}$, thereby for $\boldsymbol{r} \in \mathbb{C}^{k}$ the mapping $\hat{V} c \boldsymbol{r}$ yields a vector in $\mathcal{V}$ of the form $\hat{V} c \boldsymbol{r}=c \sum_{i=1}^{k} v_{i}\left\langle\boldsymbol{e}_{i}, \boldsymbol{r}\right\rangle$. Note that the matrix representation of $\hat{W}^{*} \hat{V}$ has elements given by the inner products $\left\langle u_{i}, v_{j}\right\rangle, i, j=1, \ldots, k$. The operator

$$
\hat{V}\left(\hat{W}^{*} \hat{V}\right)^{\dagger} \hat{W}^{*}
$$

where $(\cdot)^{\dagger}$ denotes the Moore-Penrose pseudo-inverse, is known to be the oblique projector onto $\mathcal{V}$ along $\mathcal{W}^{\perp}$ [15]. The particular choice

$$
u_{i}=v_{i}-\hat{P}_{\mathcal{W} \perp} v_{i}=\hat{P}_{\mathcal{W}} v_{i}, \quad i=1, \ldots, k
$$

produces the expression for $\hat{E}_{\mathcal{V} \mathcal{W}^{\perp}}$ used in signal processing applications [13]. Certainly, setting $\hat{W}=\hat{P}_{\mathcal{W}} \hat{V}$ one has the convenient equation

$$
\hat{E}_{\mathcal{V} \mathcal{W}^{\perp}}=\hat{V}\left(\hat{V}^{*} \hat{P}_{\mathcal{W}} \hat{V}\right)^{\dagger} \hat{V}^{*} \hat{P}_{\mathcal{W}}
$$

that we adopt hereafter.

Amongst the many properties of oblique projectors that have been studied we shall recall only the basic property needed for our purpose. It follows by applying $\hat{P}_{\mathcal{W}}$ on both sides of (4), i.e.,

$$
\hat{P}_{\mathcal{W}} \hat{E}_{\mathcal{V} \mathcal{W}^{\perp}}=\hat{P}_{\mathcal{W}} \hat{V}\left(\hat{V}^{*} \hat{P}_{\mathcal{W}} \hat{V}\right)^{\dagger} \hat{V}^{*} \hat{P}_{\mathcal{W}}
$$

Since $\hat{V}^{*} \hat{P}_{\mathcal{W}}^{*}=\hat{V}^{*} \hat{P}_{\mathcal{W}}$ and $\hat{V}^{*} \hat{P}_{\mathcal{W}} \hat{V}=\hat{V}^{*} \hat{P}_{\mathcal{W}}^{2} \hat{V}$, with the substitution $A=\hat{P}_{\mathcal{W}} \hat{V}$ the right hand side of (5) takes the form $\hat{A}\left(\hat{A}^{*} \hat{A}\right)^{\dagger} \hat{A}^{*}$. Such an operator is the orthogonal projector onto $\mathcal{R}(\hat{A})$. Consequently,

$$
\hat{P}_{\mathcal{W}} \hat{E}_{\mathcal{V} \mathcal{W}^{\perp}}=\hat{P}_{\mathcal{W}}, \quad \text { with } \quad \mathcal{W}=\mathcal{R}\left(\hat{P}_{\mathcal{W}} \hat{V}\right)=\operatorname{span}\left\{\hat{P}_{\mathcal{W}} v_{i}\right\}_{i=1}^{k}
$$

By denoting $\tilde{u}_{i}=\sum_{j=1}^{k} g_{i, j}^{\dagger} u_{j}$ with $g_{i, j}^{\dagger}$ the element $(i, j)$ of matrix $\left(\hat{V}^{*} \hat{P}_{\mathcal{W}} \hat{V}\right)^{\dagger}$, we can express $\hat{E}_{\mathcal{V} \mathcal{W}^{\perp} \text { as }}$

$$
\hat{E}_{\mathcal{V} \mathcal{W}^{\perp}}=\sum_{i=1}^{k} v_{i}\left\langle\tilde{u}_{i}, \cdot\right\rangle .
$$

Furthermore, from (6) $(17)$, and (3)

$$
\hat{P}_{\mathcal{W}}=\hat{P}_{\mathcal{W}} \hat{E}_{\mathcal{V} \mathcal{W}^{\perp}}=\sum_{i=1}^{k} u_{i}\left\langle\tilde{u}_{i}, \cdot\right\rangle .
$$


Because $\hat{P}_{\mathcal{W}}$ is self-adjoint $\operatorname{span}\left\{\tilde{u}_{i}\right\}_{i=1}^{k}=\operatorname{span}\left\{u_{i}\right\}_{i=1}^{k}=\mathcal{W}$, and vice versa. On comparing (77) and (8) we see that the dual vectors $\tilde{u}_{i}$ are the same. This is of enormous assistance to derive the equations for adapting oblique projectors so as to account for the updating or downdating of the projecting subspace $\mathcal{V}$. This will allow us to give the proofs of the proposed recursive equations either by verification or by induction.

Remark 1. It is appropriate to stress at this point that if we chose $\mathcal{W}^{\perp}=\mathcal{V}^{\perp}$ we would have $u_{i} \equiv v_{i}, i=1, \ldots, k$ and consequently $\operatorname{span}\left\{u_{i}\right\}_{i=1}^{k} \equiv \operatorname{span}\left\{v_{i}\right\}_{i=1}^{k}$. Hence for such special situation $\hat{E}_{\mathcal{V} \mathcal{V}^{\perp}} \equiv \hat{P}_{\mathcal{W}} \equiv \hat{P}_{\mathcal{V}}$ and all the recursive equations of the subsequent sections would give rise to orthogonal projectors.

\section{Constructing recursive equations}

In this section we provide the equations for updating and downdating oblique projectors in order to account for the following situations:

Let us consider that the oblique projector $\hat{E}_{\mathcal{V}_{k} \mathcal{W}^{\perp}}$ onto the subspace $\mathcal{V}_{k}=\operatorname{span}\left\{v_{i}\right\}_{i=1}^{k}$ along a given subspace $\mathcal{W}^{\perp}$ is known. If the subspace $\mathcal{V}_{k}$ is enlarged to $\mathcal{V}_{k+1}$ by the inclusion of one element, i.e., $\mathcal{V}_{k+1}=\operatorname{span}\left\{v_{i}\right\}_{i=1}^{k+1}$, we wish to construct $\hat{E}_{\mathcal{V}_{k+1} \mathcal{W}^{\perp}}$ from the availability of $\hat{E}_{\mathcal{V}_{k} \mathcal{W}^{\perp}}$. On the other hand, if the subspace $\mathcal{V}_{k}=\operatorname{span}\left\{v_{i}\right\}_{i=1}^{k}$ is reduced by the elimination of one element, say the $j$-th one, we wish to construct the corresponding oblique projector $\hat{E}_{\mathcal{V}_{k \backslash j} \mathcal{W}^{\perp}}$ from the knowledge of $\hat{E}_{\mathcal{V}_{k} \mathcal{W}^{\perp}}$. The subspace $\mathcal{W}^{\perp}$ is assumed to be fixed. Its orthogonal complement $\mathcal{W}_{k}$ in $\mathcal{H}_{k}=\mathcal{V}_{k}+\mathcal{W}^{\perp}$ changes with the index $k$ to satisfy $\mathcal{H}_{k}=\mathcal{W}_{k} \oplus \mathcal{W}^{\perp}$, where $\oplus$ denotes an orthogonal sum whilst the former is a direct sum, i.e., $\mathcal{V}_{k} \cap \mathcal{W}^{\perp}=\{0\}$.

\subsection{Updating the oblique projector $\hat{E}_{\mathcal{V}_{k} \mathcal{W}^{\perp}}$ to $\hat{E}_{\mathcal{V}_{k+1} \mathcal{W}^{\perp}}$}

We assume that $\hat{E}_{\mathcal{V}_{k} \mathcal{W}^{\perp}}$ is known and write it in the explicit form

$$
\hat{E}_{\mathcal{V}_{k} \mathcal{W} \perp}=\sum_{i=1}^{k} v_{i}\left\langle\tilde{u}_{i}^{k}, \cdot\right\rangle
$$

Our aim is to find the vector $\tilde{u}_{k+1}^{k+1}$, and to change the vectors $\tilde{u}_{i}^{k}, i=1, \ldots, k$ to $\tilde{u}_{i}^{k+1}, i=1, \ldots, k$, so as to obtain

$$
\hat{E}_{\mathcal{V}_{k+1} \mathcal{W}^{\perp}}=\sum_{i=1}^{k+1} v_{i}\left\langle\tilde{u}_{i}^{k+1}, \cdot\right\rangle .
$$

We will show that the duals $\tilde{u}_{i}^{k+1}, i=1, \ldots, k+1$ can be constructed inductively from the dual of a single vector.

Lemma 1. For $\tilde{u}_{1}^{1}=\frac{u_{1}}{\left\|u_{1}\right\|^{2}}$, with $u_{1}=\hat{P}_{\mathcal{W}} v_{1}$, operator $v_{1}\left\langle\tilde{u}_{1}^{1}, \cdot\right\rangle$ is the oblique projector onto the span of the single vector $v_{1}$ along $\mathcal{W}^{\perp}$.

Proof. From the definition of $u_{1}$ (C.f. eq. (3i)) it follows that the operator $v_{1}\left\langle\tilde{u}_{1}^{1}, \cdot\right\rangle=v_{1}\left\langle\frac{u_{1}}{\left\|u_{1}\right\|^{2}}, \cdot\right\rangle$ maps every vector in $\mathcal{W}^{\perp}$ to the zero vector. Suppose that $f$ is in the span of $v_{1}$. Then $f=c v_{1}$ for some constant $c$. Since $\left\langle u_{1}, u_{1}\right\rangle=\left\langle u_{1}, v_{1}-\hat{P}_{\mathcal{W}^{\perp}} v_{1}\right\rangle=\left\langle u_{1}, v_{1}\right\rangle$ we have

$$
v_{1}\left\langle\tilde{u}_{1}^{1}, c f\right\rangle=c v_{1} \frac{\left\langle u_{1}, v_{1}\right\rangle}{\left\|u_{1}\right\|^{2}}=c v_{1}=f .
$$


Moreover $v_{1}\left\langle\tilde{u}_{1}^{1}, v_{1}\right\rangle\left\langle\tilde{u}_{1}^{1}, \cdot\right\rangle=v_{1}\left\langle\tilde{u}_{1}^{1}, \cdot\right\rangle$, which concludes the proof that $v_{1}\left\langle\tilde{u}_{1}^{1}, \cdot\right\rangle$ is the oblique projector onto the span of $v_{1}$ along $\mathcal{W}^{\perp}$.

In order to inductively construct from $\tilde{u}_{1}^{1}=\frac{u_{1}}{\left\|u_{1}\right\|^{2}}$ the duals $\tilde{u}_{i}^{k+1}, i=1, \ldots, k+1$ we have to discriminate two possibilities

i) $\mathcal{V}_{k+1}=\operatorname{span}\left\{v_{i}\right\}_{i=1}^{k+1}=\operatorname{span}\left\{v_{i}\right\}_{i=1}^{k}=\mathcal{V}_{k}$, i.e., $v_{k+1} \in \mathcal{V}_{k}$.

ii) $\mathcal{V}_{k+1}=\operatorname{span}\left\{v_{i}\right\}_{i=1}^{k+1} \supset \operatorname{span}\left\{v_{i}\right\}_{i=1}^{k}=\mathcal{V}_{k}$, i.e. $v_{k+1} \notin \mathcal{V}_{k}$

Let us consider first the case i). Clearly if $v_{k+1} \in \mathcal{V}_{k}$ the corresponding $u_{k+1}=v_{k+1}-\hat{P}_{\mathcal{W} \perp} v_{k+1}$ belongs to $\mathcal{W}_{k}=\operatorname{span}\left\{u_{i}\right\}_{i=1}^{k}$, because $v_{k+1}=\sum_{i=1}^{k} c_{i} v_{i}$ yields $u_{k+1}=\sum_{i=1}^{k} c_{i} u_{i}$. The proposition below prescribes how to modify the corresponding dual vectors in order to guarantee that $\hat{E}_{\mathcal{V}_{k+1} \mathcal{W}^{\perp}}=\hat{E}_{\mathcal{V}_{k} \mathcal{W}^{\perp}}$

Proposition 1. Let $v_{k+1} \in \mathcal{V}_{k}$ and vectors $\tilde{u}_{i}^{k}$ in (9) be given. For an arbitrary vector $y_{k+1} \in \mathcal{H}$ the dual vectors $\tilde{u}_{i}^{k+1}$ computed as

$$
\tilde{u}_{i}^{k+1}=\tilde{u}_{i}^{k}-\left\langle u_{k+1}, \tilde{u}_{i}^{k}\right\rangle y_{k+1}
$$

for $i=1, \ldots, k$ and $\tilde{u}_{k+1}^{k+1}=y_{k+1}$ produce the identical oblique projector as the dual vectors $\tilde{u}_{i}^{k}, i=1, \ldots, k$.

Proof. We use (11) to explicitly express $\hat{E}_{\mathcal{V}_{k+1} \mathcal{W}^{\perp}}$

$$
\begin{aligned}
\sum_{i=1}^{k+1} v_{i}\left\langle\tilde{u}_{i}^{k+1}, \cdot\right\rangle & =\sum_{i=1}^{k} v_{i}\left\langle\tilde{u}_{i}^{k}, \cdot,\right\rangle-\sum_{i=1}^{k} v_{i}\left\langle\tilde{u}_{i}^{k}, u_{k+1}\right\rangle\left\langle y_{k+1}, \cdot\right\rangle+v_{k+1}\left\langle y_{k+1}, \cdot\right\rangle \\
& =\hat{E}_{\mathcal{V}_{k} \mathcal{W} \perp}-\hat{E}_{\mathcal{V}_{k} \mathcal{W} \perp} u_{k+1}\left\langle y_{k+1}, \cdot\right\rangle+v_{k+1}\left\langle y_{k+1}, \cdot\right\rangle \\
& =\hat{E}_{\mathcal{V}_{k} \mathcal{W} \perp}-v_{k+1}\left\langle y_{k+1}, \cdot\right\rangle+v_{k+1}\left\langle y_{k+1}, \cdot\right\rangle,
\end{aligned}
$$

where the last equality holds because $\hat{E}_{\mathcal{V}_{k} \mathcal{W}^{\perp}} \hat{P}_{\mathcal{W}^{\perp}}=0$ and $\hat{E}_{\mathcal{V}_{k} \mathcal{W}} v_{k+1}=v_{k+1}$ for $v_{k+1} \in \mathcal{V}_{k}$. Hence, the left hand side of (12) equals $\hat{E}_{\mathcal{V}_{k} \mathcal{W}^{\perp}}$.

The next proposition considers the case ii)

Proposition 2. Let vector $v_{k+1} \notin \mathcal{V}_{k}$ and vectors $\tilde{u}_{i}^{k}, i=1, \ldots, k$ in (9) be given. Thus the dual vectors $\tilde{u}_{i}^{k+1}$ computed as

$$
\tilde{u}_{i}^{k+1}=\tilde{u}_{i}^{k}-\tilde{u}_{k+1}^{k+1}\left\langle u_{k+1}, \tilde{u}_{i}^{k}\right\rangle
$$

where $\tilde{u}_{k+1}^{k+1}=\frac{q_{k+1}}{\left\|q_{k+1}\right\|^{2}}$ with $q_{k+1}=u_{k+1}-\hat{P}_{\mathcal{W}_{k}} u_{k+1}$, provide us with the oblique projector $\hat{E}_{\mathcal{V}_{k+1} \mathcal{W}^{\perp}}$.

Proof. In order to organize the proof let us establish the following relations:

$$
\begin{aligned}
\left\langle q_{k+1}, v_{i}\right\rangle & =0, \quad \text { for } i=1, \ldots, k \\
\left\langle q_{k+1}, v_{k+1}\right\rangle & =\left\langle u_{k+1}, v_{k+1}\right\rangle-\left\langle v_{k+1}, \hat{P}_{\mathcal{W}} v_{k+1}\right\rangle=\left\|q_{k+1}\right\|^{2} .
\end{aligned}
$$

The first relation follows from the definition of $q_{k+1}$ and the fact that $\hat{P}_{\mathcal{W}_{k}} v_{i}=u_{i}$ for $i=1, \ldots, k$

$$
\left\langle q_{k+1}, v_{i}\right\rangle=\left\langle u_{k+1}, v_{i}\right\rangle-\left\langle\hat{P}_{\mathcal{W}_{k}} u_{k+1}, v_{i}\right\rangle=\left\langle u_{k+1}, u_{i}\right\rangle+\left\langle u_{k+1}, \hat{P}_{\mathcal{W} \perp} v_{i}\right\rangle-\left\langle u_{k+1}, \hat{P}_{\mathcal{W}_{k}} v_{i}\right\rangle=0 .
$$


On the other hand

$$
\left\langle q_{k+1}, v_{k+1}\right\rangle=\left\langle u_{k+1}, v_{k+1}\right\rangle-\left\langle u_{k+1}, \hat{P}_{\mathcal{W}_{k}} v_{k+1}\right\rangle=\left\langle u_{k+1}, v_{k+1}\right\rangle-\left\langle v_{k+1}, \hat{P}_{\mathcal{W}_{k}} v_{k+1}\right\rangle .
$$

Furthermore

$$
\begin{aligned}
\left\|q_{k+1}\right\|^{2} & =\left\langle q_{k+1}, u_{k+1}\right\rangle-\left\langle q_{k+1}, \hat{P}_{\mathcal{W}_{k}} u_{k+1}\right\rangle=\left\langle q_{k+1}, u_{k+1}\right\rangle \\
& =\left\langle q_{k+1}, v_{k+1}\right\rangle-\left\langle q_{k+1}, \hat{P}_{\mathcal{W} \perp} v_{k+1}\right\rangle=\left\langle q_{k+1}, v_{k+1}\right\rangle \\
& =\left\langle u_{k+1}, v_{k+1}\right\rangle-\left\langle v_{k+1}, \hat{P}_{\mathcal{W}_{k}} v_{k+1}\right\rangle .
\end{aligned}
$$

We are now in a position to start the proof of the proposition by induction. From Lemma 1 we know that $v_{1}\left\langle u_{1}^{1}, \cdot\right\rangle /\left\|u_{1}^{1}\right\|^{2}$ is the oblique projector onto $\mathcal{V}_{1}$ along $\mathcal{W}^{\perp}$. Assuming that $\hat{E}_{\mathcal{V}_{k} \mathcal{W}^{\perp}}$ is the oblique projector onto $\mathcal{V}_{k}$ along $\mathcal{W}^{\perp}$ we will prove that $\hat{E}_{\mathcal{V}_{k+1} \mathcal{W} \perp}$ is the oblique projector onto $\mathcal{V}_{k+1}$ along $\mathcal{W}^{\perp}$. For this we need to prove that the recursive equation (13) yields the

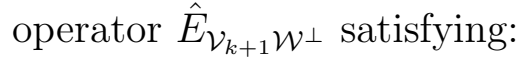

i) $\hat{E}_{\mathcal{V}_{k+1} \mathcal{W}^{\perp}}^{2}=\hat{E}_{\mathcal{V}_{k+1} \mathcal{W}^{\perp}}$

ii) $\hat{E}_{\mathcal{V}_{k+1} \mathcal{W}} v=v$, for any $v \in \mathcal{V}_{k+1}$

iii) $\hat{E}_{\mathcal{V}_{k+1} \mathcal{W}^{\perp}} w=0, \quad$ for any $\quad w \in \mathcal{W}^{\perp}$.

We begin by using (13) to express $\hat{E}_{\mathcal{V}_{k+1} \mathcal{W}^{\perp}}$ as

$$
\begin{aligned}
\sum_{i=1}^{k+1} v_{i}\left\langle\tilde{u}_{i}^{k+1}, \cdot\right\rangle & =\sum_{i=1}^{k} v_{i}\left\langle\tilde{u}_{i}^{k}, \cdot,\right\rangle-\sum_{i=1}^{k} v_{i}\left\langle\tilde{u}_{i}^{k}, u_{k+1}\right\rangle\left\langle\tilde{u}_{k+1}^{k+1}, \cdot\right\rangle+v_{k+1}\left\langle\tilde{u}_{k+1}^{k+1}, \cdot\right\rangle \\
& =\hat{E}_{\mathcal{V}_{k} \mathcal{W} \perp}-\hat{E}_{\mathcal{V}_{k} \mathcal{W} \perp} u_{k+1}\left\langle\frac{q_{k+1}}{\left\|q_{k+1}\right\|^{2}}, \cdot\right\rangle+v_{k+1}\left\langle\frac{q_{k+1}}{\left\|q_{k+1}\right\|^{2}}, \cdot\right\rangle
\end{aligned}
$$

For all $w$ in $\mathcal{W}^{\perp}$ it holds that $\hat{E}_{\mathcal{V}_{k} \mathcal{W} \perp} w=0$ and $\left\langle q_{k+1}, w\right\rangle=0$. Then from (16) we conclude that condition iii) is satisfied. Every $v \in \mathcal{V}_{k+1}$ can be written as $v=\sum_{i=1}^{k+1} c_{i} v_{i}$. Thus, from (16) and using relations (14) and (15)

$$
\begin{aligned}
\hat{E}_{\mathcal{V}_{k+1} \mathcal{W}} v & =\sum_{i=1}^{k} c_{i} v_{i}+c_{k+1} \hat{E}_{\mathcal{V}_{k} \mathcal{W}} v_{k+1}-c_{k+1} \hat{E}_{\mathcal{V}_{k} \mathcal{W} \perp} u_{k+1}+c_{k+1} v_{k+1} \\
& =\sum_{i=1}^{k} c_{i} v_{i}+c_{k+1} v_{k+1}=v
\end{aligned}
$$

which demonstrates condition ii). Finally, since from (16) and (14) it follows that $\hat{E}_{\mathcal{V}_{k+1} \mathcal{W}^{\perp}} \hat{E}_{\mathcal{V}_{k} \mathcal{W}^{\perp}}=$ $\hat{E}_{\mathcal{V}_{k} \mathcal{W}^{\perp}, \text { we have }}$

$$
\begin{aligned}
\hat{E}_{\mathcal{V}_{k+1} \mathcal{W}^{\perp}}^{2} & =\hat{E}_{\mathcal{V}_{k} \mathcal{W}^{\perp}}-\hat{E}_{\mathcal{V}_{k} \mathcal{W}^{\perp}} u_{k+1}\left\langle\frac{q_{k+1}}{\left\|q_{k+1}\right\|^{2}}, \cdot\right\rangle+\hat{E}_{\mathcal{V}_{k+1} \mathcal{W}^{\perp} v_{k+1}}\left\langle\frac{q_{k+1}}{\left\|q_{k+1}\right\|^{2}}, \cdot\right\rangle \\
& =\hat{E}_{\mathcal{V}_{k} \mathcal{W}^{\perp}}-\hat{E}_{\mathcal{V}_{k} \mathcal{W}^{\perp}} u_{k+1}\left\langle\frac{q_{k+1}}{\left\|q_{k+1}\right\|^{2}}, \cdot\right\rangle+v_{k+1}\left\langle\frac{q_{k+1}}{\left\|q_{k+1}\right\|^{2}}, \cdot\right\rangle=\hat{E}_{\mathcal{V}_{k+1} \mathcal{W}^{\perp}}
\end{aligned}
$$


Property 1. If vectors $\left\{v_{i}\right\}_{i=1}^{k}$ are linearly independent they are also biorthogonal to the dual vectors arising inductively from the recursive equation (13).

The proof of this property is given in Appendix A.

Remark 2. If vectors $\left\{v_{i}\right\}_{i=1}^{k}$ are not linearly independent the oblique projector $\hat{E}_{\mathcal{V}_{k} \mathcal{W}^{\perp}}$ is not unique. Indeed, if $\left\{\tilde{u}_{i}^{k}\right\}_{i=1}^{k}$ are dual vectors giving rise to $\hat{E}_{\mathcal{V}_{k} \mathcal{W}^{\perp}}$ then one can construct infinitely many duals as:

$$
\tilde{y}_{i}=\tilde{u}_{i}^{k}+y_{i}-\sum_{j=1}^{k} y_{j}\left\langle v_{j}, \tilde{u}_{i}^{k}\right\rangle \quad i=1, \ldots, k,
$$

where $y_{i}, i=1, \ldots, k$ are arbitrary vectors in $\mathcal{H}$.

Proof. We use (18) to write

$$
\begin{aligned}
\sum_{i=1}^{k} v_{i}\left\langle\tilde{y}_{i}, \cdot\right\rangle & =\hat{E}_{\mathcal{V}_{k} \mathcal{W}^{\perp}}+\sum_{i=1}^{k} v_{i}\left\langle y_{i}, \cdot\right\rangle-\sum_{i=1}^{k} v_{i} \sum_{j=1}^{k}\left\langle\tilde{u}_{i}^{k}, v_{j}\right\rangle\left\langle y_{j}, \cdot\right\rangle \\
& =\hat{E}_{\mathcal{V}_{k} \mathcal{W}^{\perp}}+\sum_{i=1}^{k} v_{i}\left\langle y_{i}, \cdot\right\rangle-\sum_{j=1}^{k} \sum_{i=1}^{k} v_{i}\left\langle\tilde{u}_{i}^{k}, v_{j}\right\rangle\left\langle y_{j}, \cdot\right\rangle \\
& =\hat{E}_{\mathcal{V}_{k} \mathcal{W}^{\perp}}+\sum_{i=1}^{k} v_{i}\left\langle y_{i}, \cdot\right\rangle-\sum_{j=1}^{k} v_{j}\left\langle y_{j}, \cdot\right\rangle \\
& =\hat{E}_{\mathcal{V}_{k} \mathcal{W}^{\perp}} .
\end{aligned}
$$

It follows from Property 1 that if vectors $\left\{v_{i}\right\}_{i=1}^{k}$ are linearly independent equation (18) yields the unique duals $\tilde{y}_{i} \equiv \tilde{u}_{i}^{k}, i=1, \ldots, k$.

\subsection{Downdating the oblique projector $\hat{E}_{\mathcal{V}_{k} \mathcal{W}^{\perp}}$ to $\hat{E}_{\mathcal{V}_{k j j} \mathcal{W}^{\perp}}$}

Suppose that by the elimination of the element $j$ the subspace $\mathcal{V}_{k}$ is reduced to $\mathcal{V}_{k \backslash j}=$ $\operatorname{span}\left\{v_{i}\right\}_{\substack{i=1 \\ i \neq j}}^{k}$. In order to give the equations for adapting the corresponding dual vectors generating the oblique projector $\hat{E}_{\mathcal{V}_{k \backslash j} \mathcal{W} \perp}$ we need to consider two situations:

i) $\mathcal{V}_{k \backslash j}=\operatorname{span}\left\{v_{i}\right\}_{\substack{i=1 \\ i \neq j}}^{k}=\operatorname{span}\left\{v_{i}\right\}_{i=1}^{k}=\mathcal{V}_{k}$ i.e., $v_{j} \in \mathcal{V}_{k \backslash j}$

ii) $\mathcal{V}_{k \backslash j}=\operatorname{span}\left\{v_{i}\right\}_{\substack{i=1 \\ i \neq j}}^{k} \subset \operatorname{span}\left\{v_{i}\right\}_{i=1}^{k}=\mathcal{V}_{k}$, i.e., $v_{j} \notin \mathcal{V}_{k \backslash j}$.

The next proposition addresses i).

Proposition 3. Let $\hat{E}_{\mathcal{V}_{k} \mathcal{W}}$ be given by (9) and let us assume that removing vector $v_{j}$ from the spanning set of $\mathcal{V}_{k}$ leaves the identical subspace, i.e., $v_{j} \in \mathcal{V}_{k \backslash j}$. Hence, if the remaining dual vectors are modified as follows:

$$
\tilde{u}_{i}^{k \backslash j}=\tilde{u}_{i}^{k}+\frac{\left\langle u_{j}, \tilde{u}_{i}^{k}\right\rangle \tilde{u}_{j}^{k}}{1-\left\langle u_{j}, \tilde{u}_{j}^{k}\right\rangle},
$$

the corresponding oblique projector does not change, i.e. $\hat{E}_{\mathcal{V}_{k \backslash j} \mathcal{W} \perp}=\hat{E}_{\mathcal{V}_{k} \mathcal{W} \perp}$. 
Proof. Let us recall that $v_{j} \in \mathcal{V}_{k \backslash j}$ implies $u_{j} \in \mathcal{V}_{k \backslash j}$. Hence $\left\langle u_{j}, \tilde{u}_{j}^{k}\right\rangle \neq 1$, as it is seen from the fact that $\left\langle u_{j}, \tilde{u}_{j}^{k}\right\rangle=\sum_{i=1}^{k}\left\langle u_{j}, \tilde{u}_{i}^{k}\right\rangle\left\langle u_{i}, \tilde{u}_{j}^{k}\right\rangle=\sum_{i=1}^{k}\left\langle u_{j}, \tilde{u}_{i}^{k}\right\rangle\left\langle\tilde{u}_{i}^{k}, u_{j}\right\rangle=\sum_{i=1}^{k}\left|\left\langle u_{j}, \tilde{u}_{i}^{k}\right\rangle\right|^{2}$, which implies $\left\langle u_{j}, \tilde{u}_{j}^{k}\right\rangle=1$ if and only if for $i=1, \ldots, k$ it holds that $\left\langle u_{j}, \tilde{u}_{i}^{k}\right\rangle=\delta_{i, j}$. This is not true if $v_{j} \in \mathcal{V}_{k \backslash j}$ so that we can use (20) to express $\hat{E}_{\mathcal{V}_{k \backslash j} \mathcal{W} \perp}$ as

$$
\begin{aligned}
& \sum_{\substack{i=1 \\
i \neq j}}^{k} v_{i}\left\langle\tilde{u}_{i}^{k \backslash j}, \cdot\right\rangle=\sum_{\substack{i=1 \\
i \neq j}}^{k} v_{i}\left\langle\tilde{u}_{i}^{k}, \cdot\right\rangle+\sum_{\substack{i=1 \\
i \neq j}}^{k} \frac{v_{i}\left\langle\tilde{u}_{i}^{k}, u_{j}\right\rangle\left\langle\tilde{u}_{j}^{k}, \cdot\right\rangle}{1-\left\langle\tilde{u}_{j}^{k}, u_{j}\right\rangle} \\
& =\hat{E}_{\mathcal{V}_{k} \mathcal{W}^{\perp}}-v_{j}\left\langle\tilde{u}_{j}^{k}, \cdot\right\rangle+\frac{\hat{E}_{\mathcal{V}_{k} \mathcal{W} \perp} u_{j}\left\langle\tilde{u}_{j}^{k}, \cdot\right\rangle}{1-\left\langle\tilde{u}_{j}^{k}, u_{j}\right\rangle}-v_{j}\left\langle\tilde{u}_{j}^{k}, u_{j}\right\rangle \frac{\left\langle\tilde{u}_{j}^{k}, \cdot\right\rangle}{1-\left\langle\tilde{u}_{j}^{k}, u_{j}\right\rangle} \\
& =\hat{E}_{\mathcal{V}_{k} \mathcal{W}^{\perp}}-v_{j}\left\langle\tilde{u}_{j}^{k}, \cdot\right\rangle+\frac{v_{j}\left\langle\tilde{u}_{j}^{k}, \cdot\right\rangle}{1-\left\langle\tilde{u}_{j}^{k}, u_{j}\right\rangle}-\frac{v_{j}\left\langle\tilde{u}_{j}^{k}, \cdot\right\rangle}{1-\left\langle\tilde{u}_{j}^{k}, u_{j}\right\rangle}\left\langle\tilde{u}_{j}^{k}, u_{j}\right\rangle \\
& =\hat{E}_{\mathcal{V}_{k} \mathcal{W}^{\perp}} \text {. }
\end{aligned}
$$

Finally, proposition 4 addresses ii).

Proposition 4. Let $\hat{E}_{\mathcal{V}_{k} \mathcal{W} \perp}$ be given by (91) and let us assume that the vector $v_{j}$ to be removed from the spanning set of $\mathcal{V}_{k}$ is not in $\mathcal{V}_{k \backslash j}$. In order to produce the oblique projector $\hat{E}_{\mathcal{V}_{k \backslash j} \mathcal{W}^{\perp} \text { the }}$ appropriate modification of the dual vectors can be achieved by means of the following equation

$$
\tilde{u}_{i}^{k \backslash j}=\tilde{u}_{i}^{k}-\frac{\tilde{u}_{j}^{k}\left\langle\tilde{u}_{j}^{k}, \tilde{u}_{i}^{k}\right\rangle}{\left\|\tilde{u}_{j}^{k}\right\|^{2}} .
$$

Proof. Using (22) we write:

$$
\begin{aligned}
& \sum_{\substack{i=1 \\
i \neq j}}^{k} v_{i}\left\langle\tilde{u}_{i}^{k \backslash j}, \cdot\right\rangle=\sum_{\substack{i=1 \\
i \neq j}}^{k} v_{i}\left\langle\tilde{u}_{i}^{k}, \cdot\right\rangle-\sum_{\substack{i=1 \\
i \neq j}}^{k} \frac{v_{i}\left\langle\tilde{u}_{i}^{k}, \tilde{u}_{j}^{k}\right\rangle\left\langle\tilde{u}_{j}^{k}, \cdot\right\rangle}{\left\|\tilde{u}_{j}^{k}\right\|^{2}} \\
& =\hat{E}_{\mathcal{V}_{k} \mathcal{W}^{\perp}}-v_{j}\left\langle\tilde{u}_{j}^{k}, \cdot\right\rangle-\frac{\hat{E}_{\mathcal{V}_{k} \mathcal{W} \perp} \tilde{u}_{j}^{k}\left\langle\tilde{u}_{j}^{k}, \cdot\right\rangle}{\left\|\tilde{u}_{j}^{k}\right\|^{2}}+v_{j}\left\langle\tilde{u}_{j}^{k}, \cdot\right\rangle \\
& =\hat{E}_{\mathcal{V}_{k} \mathcal{W} \perp}-\frac{\hat{E}_{\mathcal{V}_{k} \mathcal{W} \perp} \tilde{u}_{j}^{k}\left\langle\tilde{u}_{j}^{k}, \cdot\right\rangle}{\left\|\tilde{u}_{j}^{k}\right\|^{2}} .
\end{aligned}
$$

We notice that $\frac{\tilde{u}_{j}^{k}\left\langle\tilde{u}_{j}^{k}, \cdot\right\rangle}{\left\|\tilde{u}_{j}^{k}\right\|^{2}}$ is the orthogonal projector onto the span of the single vector $\tilde{u}_{j}^{k}$ and denote it as $\hat{P}_{\tilde{u}_{j}^{k}}=\frac{\tilde{u}_{j}^{k}\left\langle\tilde{u}_{j}^{k} \cdot \cdot\right\rangle}{\left\|\tilde{u}_{j}^{k}\right\|^{2}}$. Thus the orthogonal projector onto $\mathcal{W}_{k \backslash j}$ can be expressed as $\hat{P}_{\mathcal{W}_{k \backslash j}}=\hat{P}_{\mathcal{W}_{k}}-\hat{P}_{\tilde{u}_{j}^{k}}$. Applying this operator on the right hand side of (23) we obtain:

$$
\hat{P}_{\mathcal{W}_{k \backslash j}}\left(\hat{E}_{\mathcal{V}_{k} \mathcal{W} \perp}-\hat{E}_{\mathcal{V}_{k} \mathcal{W} \perp} \hat{P}_{\tilde{u}_{j}^{k}}\right)=\left(\hat{P}_{\mathcal{W}_{k}}-\hat{P}_{\tilde{u}_{j}^{k}}\right)\left(\hat{E}_{\mathcal{V}_{k} \mathcal{W} \perp}-\hat{E}_{\mathcal{V}_{k} \mathcal{W} \perp} \hat{P}_{\tilde{u}_{j}^{k}}\right)
$$

and, since $\hat{P}_{\mathcal{W}_{k}} \hat{E}_{\mathcal{V}_{k} \mathcal{W} \perp}=\hat{P}_{\mathcal{W}_{k}}$ and $\hat{P}_{\tilde{u}_{j}^{k}} \hat{P}_{\mathcal{W}_{k}}=\hat{P}_{\tilde{u}_{j}^{k}}$, using the fact that $\hat{P}_{\tilde{u}_{j}^{k}} \hat{E}_{\mathcal{V}_{k} \mathcal{W} \perp}=\hat{P}_{\tilde{u}_{j}^{k}} \hat{P}_{\mathcal{W}_{k}} \hat{E}_{\mathcal{V}_{k} \mathcal{W} \perp}=$ $\hat{P}_{\tilde{u}_{j}^{k}}$ it follows that

$$
\begin{aligned}
\left(\hat{P}_{\mathcal{W}_{k}}-\hat{P}_{\tilde{u}_{j}^{k}}\right)\left(\hat{E}_{\mathcal{V}_{k} \mathcal{W}^{\perp}}-\hat{E}_{\mathcal{V}_{k} \mathcal{W}^{\perp}} \hat{P}_{\tilde{u}_{j}^{k}}\right) & =\hat{P}_{\mathcal{W}_{k}}-\hat{P}_{\tilde{u}_{j}^{k}}-\hat{P}_{\tilde{u}_{j}^{k}}+\hat{P}_{\tilde{u}_{j}^{k}} \\
& =\hat{P}_{\mathcal{W}_{k}}-\hat{P}_{\tilde{u}_{j}^{k}}=\hat{P}_{\mathcal{W}_{k \backslash j}}=\hat{P}_{\mathcal{W}_{k \backslash j}} \hat{E}_{\mathcal{V}_{k \backslash j} \mathcal{W}} .
\end{aligned}
$$


From the last equation and (23) we gather that

$$
\hat{P}_{\mathcal{W}_{k \backslash j}} \sum_{\substack{i=1 \\ i \neq j}}^{k} v_{i}\left\langle\tilde{u}_{i}^{k \backslash j}, \cdot\right\rangle-\hat{P}_{\mathcal{W}_{k \backslash j}} \hat{E}_{\mathcal{V}_{k \backslash j} \mathcal{W} \perp}=0 .
$$

For every vector $f \in \mathcal{H}$ we therefore have

$$
\hat{P}_{\mathcal{W}_{k \backslash j}}\left(\sum_{\substack{i=1 \\ i \neq j}}^{k} v_{i}\left\langle\tilde{u}_{i}^{k \backslash j}, \cdot\right\rangle-\hat{E}_{\mathcal{V}_{k \backslash j} \mathcal{W}^{\perp}}\right) f=\hat{P}_{\mathcal{W}_{k \backslash j}} \Delta \hat{D} f=0
$$

with $\Delta \hat{D}=\sum_{\substack{i=1 \\ i \neq j}}^{k} v_{i}\left\langle\tilde{u}_{i}^{k \backslash j}, \cdot\right\rangle-\hat{E}_{\mathcal{V}_{k \backslash j} \mathcal{W} \perp}$. This implies that either $\Delta \hat{D}$ is the zero operator or $\Delta \hat{D} f \in \mathcal{W}^{\perp}$ for every $f \in \mathcal{H}$. The latter cannot be true because from the definition of $\Delta \hat{D}$ it is seen that $\Delta \hat{D} f \in \mathcal{V}_{k \backslash j}$ and by hypothesis $\mathcal{V}_{k \backslash j} \cap \mathcal{W}^{\perp}=\{0\}$. Hence $\Delta \hat{D}$ should be the zero operator, which leads to the conclusion that

$$
\hat{E}_{\mathcal{V}_{k \backslash j} \mathcal{W} \perp}=\sum_{\substack{i=1 \\ i \neq j}}^{k} v_{i}\left\langle\tilde{u}_{i}^{k \backslash j}, \cdot\right\rangle
$$

Remark 3. The case of replacing a vector in $\mathcal{V}_{k}$, say $v_{j}$ by $v_{j}^{\prime}$, is actually equivalent to augmenting the subspace $\mathcal{V}_{k \backslash j}$ to $\mathcal{V}_{k \backslash j}+v_{j}^{\prime}$ after the vector $v_{j}$ was deleted. Some implementation issues arise, though. In order to modify the duals as prescribed in (13) we need to compute a vector $q_{k \backslash j}=u_{j}^{\prime}-\hat{P}_{\mathcal{W}_{k \backslash j}} u_{j}^{\prime}$. For the sequential enlargement of the projecting subspace, discussed in section 3.1, the projector $\hat{P}_{\mathcal{W}_{k}}$ can be sequentially constructed by means of the orthonormal vectors $q_{n} /\left\|q_{n}\right\|, n=1, \ldots, k$. Nevertheless, when replacing vectors sequentially we need to allow for the recalculation of the corresponding projectors. One possibility that could be considered is the recalculation of the orthogonal vectors $q_{n}$ [27, 28]. An alternative approach implies to use of the dual corresponding to the deleted vector for orthogonalization purposes. A discussion concerning the implementation of such a procedure is given in [29,30].

\section{Applications to signals discrimination}

The examples presented in this section aim at illustrating the application of our recursive construction of oblique projectors for signals in $L_{2}[a, b]$, the space of square integrable functions on $[a, b]$. For $f$ and $g$ in $L_{2}[a, b]$, we define the inner product, according to the previously adopted convention, as

$$
\langle f, g\rangle=\int_{a}^{b} f^{*}(x) g(x) d x
$$

where $f^{*}(x)$ indicates the complex conjugate of $f$. In the examples bellow all the integrals are numerically calculated. 


\subsection{Extraction of a X-ray diffraction peak from a dispersive back- ground}

Here the signal is simulated by emulating a crystallographic problem. It is assumed to be the X-ray diffraction intensity produced by a powder sample of a clay mineral consisting of very flat crystals. Each such crystal is formed by the stacking of $n$ layers producing a diffraction intensity as given by $[31,32]$

$$
I_{n}(x)=\frac{\sin ^{2} n x}{\sin ^{2} x}
$$

where the variable $x$, given in radians, is related to the diffraction angle $\theta$ according to the equation

$$
x=2 \pi \frac{d}{\lambda} \sin \theta
$$

The parameter $d$ in (28) is the effective distance between two consecutive layers and characterizes the material. The parameter $\lambda$ represents the wavelength of the incident radiation.

We denote the diffraction intensity produced by the whole sample as $f_{1}(x)$. Thus,

$$
f_{1}(x)=\sum_{n=1}^{k} c_{n} \frac{\sin ^{2} n x}{\sin ^{2} x} .
$$

As already mentioned $n$ indicates the possible number of layers forming a single crystal in the sample. The coefficients $c_{n}$ account for the proportions of crystals consisting of $n$ layers. Here, for simulating the signal, the coefficients were considered to be

$$
c_{n}=e^{-0.05(n-7)^{2}}+0.2 e^{-0.1(n-35)^{2}}, n=1 \ldots, 60 .
$$

The diffraction figure $f_{1}$ emerges from a background that is modelled as

$$
f_{2}(x)=50 \sum_{j=1}^{3} j e^{-j\left(x-\frac{\pi}{2}\right)} .
$$

The combined phenomenon gives rise to the signal $f=f_{1}+f_{2}$ plotted in the left graph of Figure 1 on the interval relevant to the diffraction model, namely $\left[\frac{\pi}{2}, \frac{3 \pi}{2}\right]$.

We are interested in extracting the diffraction peak $f_{1}$ from the background. For this we will construct sequentially oblique projectors onto subspaces $\mathcal{V}_{k}$ given as

$$
\mathcal{V}_{k}=\operatorname{span}\left\{\frac{\sin ^{2} n x}{\sin ^{2} x}, n=1, \ldots, k\right\}, x \in\left[\frac{\pi}{2}, \frac{3 \pi}{2}\right] .
$$

The final $k$-value is to be adjusted. The subspace $\mathcal{W}^{\perp}$ is here

$$
\mathcal{W}^{\perp}=\operatorname{span}\left\{e^{-j\left(x-\frac{\pi}{2}\right)}, j=1, \ldots, 3\right\}, x \in\left[\frac{\pi}{2}, \frac{3 \pi}{2}\right] .
$$

Since the order $k$ of the diffraction model is assumed unknown, it was adjusted as follows: firstly the order model was sequentially increased (up to $k=200$ ) and then sequentially downdated. It was observed that the recovering of the signal was not very sensitive to the model order. In a range from $k=50$ to $k=200$ the approximations were totally equivalent. From $k=40$ to $k=50$ changes in the approximations were noticed but the approximations could still be 
considered 'practically' equivalent. The recovered peak $f_{1}=\hat{E}_{\mathcal{V}_{k} \mathcal{W}} f$ (for $k=50$ ) is depicted in the right graph of Figure 1. It happens to coincide, in the scale of the figure, with the graph of the theoretical one (C.f. (29)).

The convenience of the proposed adaptive technique in the determination of the order of the diffraction intensity model is clear: otherwise when changing the $k$ value as described above the whole projector would have to be recalculated for each different value of $k$. However, the advantage of the proposed technique is even more significant when, as is the case in the next example, overestimation of the order in the signal model may result in the failure to discriminate the signals.
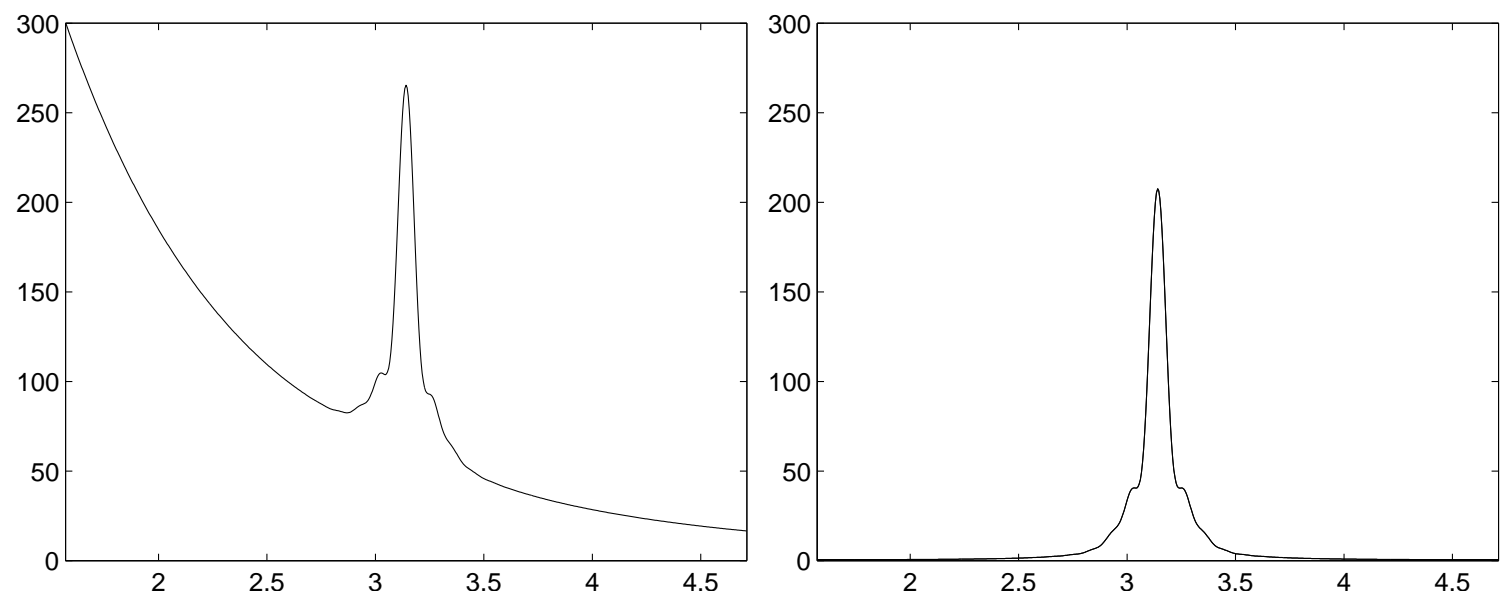

Figure 1: Graphs on the left: Diffraction intensity vs the variable $x$ measured in radians and related to the diffraction angle through (28). Graph on the right: Diffraction intensity extracted from the background.

\subsection{Elimination of impulsive noise}

In this case the signal is considered to be the register of the motion of a system consisting of uncoupled damped harmonic oscillators. The $n$-th oscillator is characterized by a frequency of $\frac{n}{2} \mathrm{~Hz}$. The corresponding equation for its motion $x_{n}(t)$ as a function of time is given as

$$
x_{n}(t)=e^{-t} \cos (\pi n t), \quad n=1, \ldots, k .
$$

The distribution of frequencies is considered to be $c_{n}=\left(1+0.7(n-75)^{2}\right)^{-1}$ so that the motion of the system is registered by the signal

$$
f_{1}(t)=\sum_{n=1}^{100} \frac{e^{-t} \cos (\pi n t)}{1+0.7(n-75)^{2}}, \quad t \in[0,1] .
$$

This signal (shown in the right graphs of Figure 2) is corrupted by impulsive noise, which represents a type of electrical noise appearing in some practical situations. The possible pulses are taking from the set of 400 Gaussian sparks $p_{j}(t)=e^{-100000(t-0.0025 j)^{2}}, j=1, \ldots, 400$. Hence the corresponding subspace $\mathcal{W}^{\perp}$ is

$$
\mathcal{W}^{\perp}=\operatorname{span}\left\{e^{-100000(t-0.0025 j)^{2}}, j=1, \ldots, 400\right\}, \quad t \in[0,1] .
$$


First a random superposition of 50 pulses is added to the signal $f_{1}$ to simulate the noisy one plotted in the top left graph of Figure 2. The result after the oblique projections along the subspace $\mathcal{W}^{\perp}$ given above is shown in the top right graph (it coincides with the theoretical signal $f_{1}$ given in (32) ). The left graph at the bottom of Figure 2 corresponds to a different realization of the noise, in this case generated as a random superposition of 200 pulses. The signal, after filtering by oblique projections along $\mathcal{W}^{\perp}$, is shown in the right graph (it also coincides with the theoretical one).
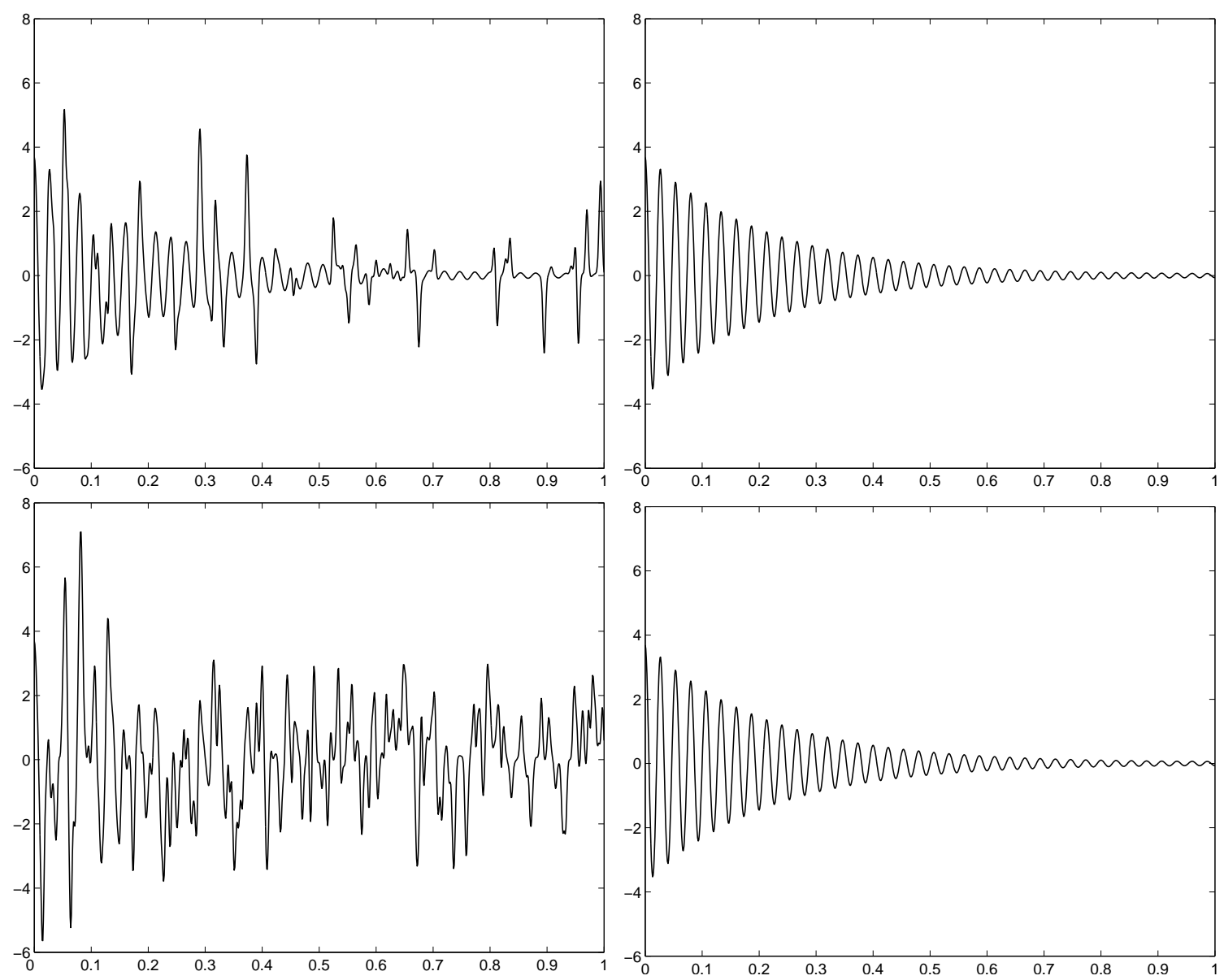

Figure 2: Top left graph: motion of the harmonic oscillators system as a function of time (in seconds) corrupted by 50 random pulses. The graph on the right depicts the signal after filtering the noise by sequential oblique projection. The bottom figures have the same description but the noise corresponds to 200 random pulses.

Let us point out that an alternative way of splitting the signal would entail fitting both the signal and noise models, with the consequent increment in the dimension of the problem of determining the corresponding unknown parameters. In the example of this section, for instance, 400 more parameters (coefficients of the noise model) would be involved. 


\section{Conclusions}

Recursive equations for updating/downdating oblique projectors have been proposed. The updating strategy can be regarded as a generalized Gram-Schmidt like procedure for generating a sequence giving rise to oblique projectors along a fixed subspace. The downdating strategy modifies such sequence to account for the removal of some elements. The equations are of the same nature as those for producing orthogonal projectors, but involve different vectors. Orthogonal projectors arise within this framework as a particular case.

The proposed technique has been applied to the problem of discriminating signals produced by different phenomena. The applications that have been considered assume that the signal model is determined by physical considerations and only the order of the model is to be adjusted. The task of setting the right order model is facilitated by the recursive nature of the proposed equations. In the two examples considered here the signal splitting is not very sensitive to the order of the signal model. However, an important difference between the two examples is the following: while in the first example an excessive overestimation of order model (maximum possible number of layers present in a crystal) does not prevent the extraction of the diffraction peak from the background, an excessive overestimation of the order of the model in the second example (maximum possible frequency of an oscillator) may produce the failure to separate the signal from the impulsive noise. The reason being that for very high frequencies the angle between the signal subspace and the noise subspace becomes very small, which generates an ill posed problem.

The recursive feature of the proposed equations turns out to be even more important in those situations in which the signals splitting is achieved by stepwise selection of each component of the signal model. This is the subject of a recent work [33], where the present approach is shown to be of significant assistance.

\section{Acknowledgements}

Support from EPSRC (EP/D062632/1) is acknowledged. 


\section{Appendix A: Proof of Property 1}

Let us recall that if vectors $\left\{v_{i}\right\}_{i=1}^{k+1}$ are linearly independent, all the duals $\left\{\tilde{u}_{i}^{k+1}\right\}_{i=1}^{k+1}$ are generated by the recursive equation (13). We need to show that such vectors satisfy:

$$
\left\langle v_{m}, \tilde{u}_{i}^{k+1}\right\rangle=\delta_{m, i}, \quad m, i=1, \ldots, k+1 .
$$

Proof. For $k=0$ the relation holds because $\tilde{u}_{1}^{1}=\frac{u_{1}}{\left\|u_{1}\right\|^{2}}$ and $\left\|u_{1}\right\|^{2}=\left\langle v_{1}, v_{1}\right\rangle-\left\langle v_{1}, \hat{P}_{\mathcal{W}} v_{1}\right\rangle$. Therefore $\left\langle v_{1}, \tilde{u}_{1}^{1}\right\rangle=\frac{\left\langle v_{1}, v_{1}\right\rangle-\left\langle v_{1}, \hat{P}_{\mathcal{W} \perp} v_{1}\right\rangle}{\left\langle v_{1}, v_{1}\right\rangle-\left\langle v_{1}, \hat{P}_{\mathcal{W} \perp} v_{1}\right\rangle}=1$.

Assuming that for $k+1=l$ it is true that

$$
\left\langle v_{m}, \tilde{u}_{i}^{l}\right\rangle=\delta_{m, i}, \quad m, i=1, \ldots, l
$$

we will prove that

$$
\left\langle v_{m}, \tilde{u}_{i}^{l+1}\right\rangle=\delta_{m, i}, \quad m, i=1, \ldots, l+1 .
$$

For this we need to consider four different situations with regard to the indices.

I) $m=1, \ldots, l$ and $i=1, \ldots, l$.

In this case $\left\langle v_{m}, q_{l+1}\right\rangle=0$ (C.f. (14)). Hence, from the recursive equation (13), we have

$$
\left\langle v_{m}, \tilde{u}_{i}^{l+1}\right\rangle=\left\langle v_{m}, \tilde{u}_{i}^{l}\right\rangle+0=\delta_{m, i} .
$$

II) $m=l+1$ and $i=1, \ldots, l$

Now

$$
\begin{aligned}
\left\langle v_{l+1}, \tilde{u}_{i}^{l+1}\right\rangle & =\left\langle v_{l+1}, \tilde{u}_{i}^{l}\right\rangle-\frac{\left\langle v_{l+1}, q_{l+1}\right\rangle}{\left\|q_{l+1}\right\|^{2}}\left\langle v_{l+1}, \tilde{u}_{i}^{l}\right\rangle \\
& =\left\langle v_{l+1}, \tilde{u}_{i}^{l}\right\rangle \frac{\left\|q_{k+1}\right\|^{2}-\left\langle v_{l+1}, q_{l+1}\right\rangle}{\left\|q_{l+1}\right\|^{2}}
\end{aligned}
$$

so that, since $\left\langle v_{l+1}, q_{l+1}\right\rangle=\left\|q_{k+1}\right\|^{2}$ (C.f. (15)),

$$
\left\langle v_{l+1}, \tilde{u}_{i}^{l+1}\right\rangle=0 \text {. }
$$

III) $m=l+1$ and $i=l+1$.

This implies

$$
\left\langle v_{l+1}, \tilde{u}_{l+1}^{l+1}\right\rangle=\frac{\left\langle v_{l+1}, q_{l+1}\right\rangle}{\left\|q_{l+1}\right\|^{2}}=1 .
$$

IV) $m=1, \ldots, l$ and $i=1+l$.

In this case

$$
\left\langle v_{m}, \tilde{u}_{l+1}^{l+1}\right\rangle=\frac{\left\langle v_{m}, q_{l+1}\right\rangle}{\left\|q_{l+1}\right\|^{2}}=0 .
$$

From I) II) III) and VI) we conclude that

$$
\left\langle v_{m}, \tilde{u}_{i}^{l+1}\right\rangle=\delta_{m, i}, \quad m, i=1, \ldots, l+1,
$$

which proves that the vectors generated through (13) are biorthogonal to vectors $v_{m}, m=$ $1, \ldots, k+1$. 


\section{References}

[1] S. N. Afriat, Orthogonal and oblique projectors and the characteristics of pairs of vectors spaces, Proc Cambridge Philos. Soc. 53, (1957), 800-816.

[2] T. N. Greville, Solutions of the matrix equations $X A X=X$ and relations between oblique and orthogonal projectors, SIAM J. Apply Math. 26 4, (1974), 828-832.

[3] S. Kayalar, H. L. Weinet, Oblique projections: Formulas, algorithms and error bounds, Math Control Signal Systems 2, (1989), 33-45.

[4] A. Aldroubli, Oblique projections in atomic spaces, Proc. Amer. Math. Soc. 124, (1996), 2051-2060.

[5] A. Forsgren, On linear least-square problems with diagonally dominant weight matrices, SIAM J. Matrix Anal. Appl. 17, (1996), 763-788.

[6] Y. Takene, H. Yanai: On oblique projectors, Linear Algebra Appl. 289, (1999), no. 1-3, 297-310.

[7] J. Groß, G. Trenkler, Nonsingularity of the difference of two oblique projectors, SIAM J. Matrix Anal. Appl. 21, (1999), no. 2, 390-395.

[8] A. Forsgren, G Sporre, On weighed linear least-squares problems related to interior methods for convex quadratic programming, SIAM J. Matrix Anal. Appl. 23, (2001), 42-56.

[9] Y. Censor, T. Elfving, Block-iterative algorithms with diagonally scaled oblique projections for the linear feasibility problem, SIAM J. Matrix Anal. Appl. 24, (2002), no. 1, 40-58.

[10] G. Gorach, A. Maestripieri, D Stojanoff, Oblique projection and abstract splines, Journal of Approximation Theory 117, (2002), 189-206.

[11] G. Gorach, A. Maestripieri, D Stojanoff, Projections in operators ranges, Proc. Amer. Math. Soc. 134, (2005), no 3, 765-788.

[12] A. Galantai, Projectors and Projection Methods, Kluwer Academic Publishers, (2004).

[13] R. T. Behrens, L. L. Scharf, Signal processing applications of oblique projection operators, IEEE Tran. Signal Processing, 42, (1994), 1413-1424.

[14] T. Blu, M Unser, Quantitative Fourier analysis of approximation techniques. I Interpolations and projections, IEEE Tran. Signal Processing, 47, (1999), 2783-2795.

[15] Y. C. Eldar, Sampling with arbitrary sampling and reconstruction spaces and oblique dual frame vectors, J. Fourier Anal. Appl, 9 no 1, (2003), 77-96.

[16] R. Raskar, Immersive planar display using roughly aligned projectors, IEEE Virtual Reality, (2000), 109-116.

[17] G. Davis, S. Mallat, and M. Avellaneda, Adaptive greedy approximations, Const. Approx., 13, no. 1, (1997), 57-98. 
[18] L. Rebollo-Neira, D. Lowe, Optimized orthogonal matching pursuit approach, IEEE Signal Processing Letters, 9, no. 4, (2002), 137-140.

[19] R. Gribonval, M. Nielsen, Nonlinear approximation with dictionaries. I. Direct estimates, J. of Fourier Anal. and Appl., 10, no. 1, (2004), 55-71.

[20] M. Andrle, L. Rebollo-Neira, E. Sagianos, Backward-optimized orthogonal matching pursuit approach, IEEE Signal Proc. Let. 11, no.9, (2004), 705-708.

[21] L. Rebollo-Neira, Recursive bi-orthogonalisation approach and orthogonal projectors, math-ph/0209026 (2002);

L. Rebollo-Neira, New Topics in Mathematical Physics Research, Nova Science Publisher, New York, (2006), Ch. On non-orthogonal signal representation.

[22] L. Rebollo-Neira, Backward adaptive biorthogonalization, Int. J. Math. Math. Sci. 2004, no 35, (2004), 1843-1853.

[23] T. N. E. Greville, The pseudoinverse of a rectangular or singular matrix and its applications, SIAM Rev 1, no 1, (1959), 38-43.

[24] A. Ben-Israel, An iterative method for computing the generalized inverse of an arbitrary matrix, Math. Comput. 19, (1965), 452-455.

[25] R. Fletcher, A technique of orthogonalization, J. Inst. Math. Appl. 5, (1969), 162-166.

[26] S. Mohideen, V. Cherkassky, On recursive calculation of the generalized inverse of a matrix, ACM Trans. Math. Softw. 17, no 1, (1991), 130-147.

[27] L. Reichel, W. B. Gragg, Algorithm 686: FORTRAN subroutines for updating the QR decomposition., ACM Trans. Math. Softw. 16, no. 4, (1990), 369-377.

[28] A. Björck, Numerical Methods for Least Squares Problems, SIAM, Philadelphia, (1996).

[29] M. Andrle, L. Rebollo-Neira, A swapping-based refinement of orthogonal matching pursuit strategies, Signal Processing, 86, no 3, (2006), 480-495.

[30] M. Andrle, L. Rebollo-Neira, Experiments on orthogonalization by biorthogonal representations of orthogonal projectors, Journal of Computational and Applied Mathematics, In press, Corrected proof available on line (2006).

[31] H. P. Klug, L. E. Alexander, X-ray Diffraction Procedures, Wiley, (1974).

[32] R. Jenkins, Introduction to X-ray Powder Diffractometry, John Wiley \& Sons Inc, (1997).

[33] L. Rebollo-Neira, Oblique Matching Pursuit, IEEE Signal Processing Letters, in press (2007). 